\title{
Gendered Energy - Analytische Perspektiven und Potenziale der Geschlechterforschung für eine sozial-ökologische Gestaltung der Energiewende im Raum
}

\author{
Helga Kanning ${ }^{1}$ Tanja Mölders ${ }^{2}$ Sabine Hofmeister ${ }^{3}$
}

Eingegangen: 30. März 2015 / Angenommen: 12. April 2016 / Online publiziert: 25. Mai 2016

(c) Springer-Verlag Berlin Heidelberg 2016

Zusammenfassung Die Energiewende stellt einen bedeutenden Transformationsprozess dar, der Räume materiell und symbolisch verändert. Diesen Wandel nachhaltig zu gestalten, stellt eine der größten Herausforderungen auf dem Weg in eine postfossile Gesellschaft dar.

Die räumlichen Planungen müssen mit diesen Herausforderungen umgehen, das heißt, insbesondere veränderte Konstellationen zwischen Akteurinnen und Akteuren sowie die Entstehung neuer Energiesysteme, -landschaften und Raumbeziehungen in ihre Aufgabenfelder einbeziehen und ihre Instrumentarien prozedural und substanziell anpassen und weiterentwickeln. Noch befinden sich sowohl die praktische Ausgestaltung der neuen Planungsaufgaben als auch die planungswissenschaftliche Forschung zur Energiewende am Anfang.

Wir gehen davon aus, dass die Verbindung raum- und planungswissenschaftlicher Debatten zur Energiewende mit analytischen Perspektiven der nachhaltigkeitsbezogenen Geschlechterforschung besondere, noch unerschlossene Potenziale für die Gestaltung der Transformation der Energiesysteme in Richtung einer nachhaltigen Entwicklung bietet. Ausgehend von unterschiedlichen analytischen

Apl. Prof. Dr. Helga Kanning

kanning@umwelt.uni-hannover.de

Prof. Dr. Tanja Mölders

t.moelders@archland.uni-hannover.de

1 Institut für Umweltplanung, Leibniz Universität Hannover, Herrenhäuserstr. 2, 30419 Hannover, Deutschland

2 Forum für GenderKompetenz in Architektur I Landschaft I Planung, Leibniz Universität Hannover, Herrenhäuserstr. 8, 30419 Hannover, Deutschland

3 Fakultät Nachhaltigkeit, Leuphana Universität Lüneburg, Scharnhorststraße 1, 21335 Lüneburg, Deutschland
Perspektiven der sozial-ökologischen Nachhaltigkeits- und Geschlechterforschung, wonach Geschlecht als Differenz-, als Struktur-, als Prozess- sowie als epistemologische Kategorie begriffen werden kann, fragen wir danach, welche „blinden Flecken“, Gerechtigkeits- und Demokratiedefizite sowie herrschaftliche Umgangsweisen mit Natur sichtbar gemacht werden können, um so Transformationswissen für eine emanzipatorische (Um-) Gestaltung der Energiewende im Raum sowie neue Perspektiven für die raum- und planungswissenschaftliche Energieforschung zu gewinnen.

Schlüsselwörter Energiewende · Gender · Geschlechterforschung · Soziale Ökologie · Raum · Räumliche Planung · Planungswissenschaften

\section{Gendered Energy - Analytical perspectives and potentials of gender research for a social-ecological transformation of "Energiewende" in space}

\footnotetext{
Abstract The "Energiewende" (energy transition) is an important transformation process changing spaces both materially and symbolically. Making this change sustainable is one of the biggest challenges on the way to a post-fossil society.

To meet these challenges in its areas of responsibility means for spatial planning to take into account in particular changed actor constellations, the emergence of new energy systems, energy landscapes and spatial relationships. However, both the implementation of the new planning tasks as well as the planning research on energy transition are only just at the beginning. Clearly, planning instruments will have to be adapted and developed procedurally and substantially.
} 
We propose that linking the debate on the Energiewende that takes place in spatial research and planning science to analytical aspects of sustainability-related gender research will achieve a yet untapped potential for transforming energy systems towards sustainable development. Using results from gender studies and research into the social ecology of sustainability, we define gender in four ways, as differential, as structure, as process and as epistemological category. Drawing upon these concepts we ask what "blind spots", justice and democracy deficits as well as manorial handling with nature can be made visible. The aim of the paper is to gain transformation knowledge for an emancipatory (re-)design of energy transition in space as well as new prospects for planning science on energy transition.

Keywords Energy transition - Gender - Gender research . Social ecology $\cdot$ Space/Place $\cdot$ Spatial planning $\cdot$ Planning science

\section{Herausforderungen für eine sozial-ökologische Gestaltung der Energiewende im Raum}

Die Energiewende ist mit radikalen Veränderungen der materiell-energetischen und damit zusammenhängend auch der gesellschaftlichen Systeme verbunden (vgl. SRU 2011: Tz 654). Als Reaktion der Gesellschaft auf den anthropogen verursachten Klimawandel und die damit verbundenen ökologischen und sozialen Folgen wird die Energiewende in Deutschland zu einer bedeutenden Transformation, die zu einer postfossilen Gesellschaft führen soll. Doch um welche Herausforderungen geht es, wenn über den Wandel der technischen Energiesysteme und -märkte hinaus insgesamt sozial-ökologische Transformationsprozesse erfolgen sollen? Welche Herausforderungen - sowohl in analytischer als auch gestalterischer Hinsicht - stellen sich, wenn die mit der Energiewende verbundenen Transformationsprozesse in Richtung einer nachhaltigen Entwicklung ausgerichtet werden sollen?

Wir nähern uns diesen Fragen aus einer inter- und transdisziplinären Perspektive und fragen danach, ob und wie die Generierung von Systemwissen zur Energiewende mit der Gewinnung reflexiven und antizipativen Wissens (WBGU 2011: 341) verbunden werden kann, sodass es für die Ableitung planerisch-gestalterischer Aussagen, das heißt als „transformative Forschung“ (WBGU 2011: 342 f.), und für die Gestaltung der Energiewende in Richtung einer nachhaltigen Entwicklung nutzbar wird. ${ }^{1}$ Wir greifen da-

\footnotetext{
1 Transformationsforschung dient dem besseren Verständnis von Transformationsprozessen; transformative Forschung unterstützt Transformationsprozesse durch die Entwicklung von Problemlösungen (WBGU 2011: 342 f.).
}

zu heuristisch das Rahmenkonzept sozial-ökologischer Forschung auf und beziehen uns auf die dort im Konzept der gesellschaftlichen Naturverhältnisse ${ }^{2}$ angelegten Kategorien Natur und Geschlecht (Becker/Hummel/Jahn 2011; vgl. auch unten im Kapitel 3). Ebenso knüpfen wir an die Forschungsprinzipien transdisziplinärer Nachhaltigkeitsforschung an (Brand 2000), ${ }^{3}$ indem wir die Energiewende nicht nur (besser) zu verstehen suchen, sondern zudem fragen, wie wir Beiträge zu deren Gestaltung generieren können.

Dabei gehen wir von der Überlegung aus, dass die Verbindung von Raum ${ }^{4}$ und räumlicher Planung auf regionaler Ebene mit analytischen Perspektiven einer nachhaltigkeitsorientierten Geschlechterforschung besondere, noch unerschlossene Potenziale bietet. Diese könnten genutzt werden, um das Transformationsfeld Energie im Sinne einer sozial-ökologischen Transformation (Becker/Hummel/Jahn 2011: 82) zu gestalten.

Für alle vom Wissenschaftlichen Beirat der Bundesregierung Globale Umweltveränderungen (WBGU) (2011) aufgezeigten Transformationsfelder zur Nachhaltigkeit, die er auf die Bereiche Urbanisierung, Landnutzung und Energie fokussiert, ist der Raum eine mögliche Integrationsebene. Denn Raum entsteht in der Verbindung verschiedener, parallel verlaufender physisch-materieller sowie symbolischdiskursiver gesellschaftlicher Wandlungsprozesse. Anders als die auf fossilen Energien basierenden Systeme, in denen die Energiegewinnung in unseren Regionen zumeist nur punktuell in der Fläche, peripher und über die zentralen Großinfrastruktureinrichtungen, das heißt die Kraftwerke und Netze, sichtbar ist, verändern die neuen, auf erneuerbaren Energien basierenden Energiesysteme unsere Kulturlandschaften in der Fläche erheblich - sie brin-

\footnotetext{
2 Als gesellschaftliche Naturverhältnisse werden die dynamischen Beziehungsmuster zwischen Mensch, Gesellschaft und Natur bezeichnet. Das sozial-ökologische Konzept unterscheidet analytisch zwischen stofflich-materiellen und kulturell-symbolischen Beziehungsaspekten, die als miteinander vermittelt gesehen werden, jedoch nicht aufeinander reduziert werden können (Becker/Hummel/Jahn 2011: 77 f.).

3 Unter Nachhaltigkeitsforschung werden Forschungen verstanden, die sich normativ am Leitbild einer nachhaltigen Entwicklung ausrichten: Im Vordergrund stehen dabei die Gerechtigkeitsgebote in intraund intergenerationaler Perspektive sowie insbesondere das Integrationsgebot, wonach ökonomische, ökologische und sozial-kulturelle Entwicklungsziele aufeinander bezogen und in Einklang miteinander zu realisieren sind.

${ }^{4}$ Aus der dem Beitrag zugrundeliegenden Verortung in der sozialökologischen Nachhaltigkeitsforschung resultiert ein Raumverständnis, das wir - aufbauend auf der doppelseitigen Kritik an naturalistisch verkürzten Raumkonzepten („Container-Raum“) und an soziozentrisch verkürzten Raumkonzepten (der Sozialraum als gesellschaftliches Konstrukt) - als vermittlungstheoretisch positioniert anlegen: Unter ,vermittlungstheoretischen Positionen“ werden ,[...] solche Ansätze [gefasst], die ,Natur' und ,Gesellschaft' nicht dualistisch und auch nicht reduktionistisch konzeptualisieren.“ (Kropp 2002: 147; $\mathrm{zu}$ „Raum“ in vermittlungstheoretischer Perspektive vgl. FBW 2007: 32 ff. sowie Hofmeister/Scurrell 2006; Hofmeister/Scurrell 2016).
} 
gen Raum sowohl physisch-materiell als auch symbolischdiskursiv neu hervor (Gailing/Hüesker/Kern et al. 2013: 23 f.; Radtke 2015; Hofmeister/Scurrell 2016). Dezentral entstehen mit Windrädern, Sonnenkollektoren, Fotovoltaikund Biogasanlagen sowie großflächigen Maisfeldern völlig neue Kulturlandschaften als industrielle ,Energielandschaften“ (vgl. z. B. BfN/BBSR 2014a; BfN/BBSR 2014b). Dieses geschieht regional in sehr unterschiedlicher Ausprägung abhängig von den jeweiligen (natur-)räumlichen Gegebenheiten, verfügbaren (Flächen-)Ressourcen und den gesellschaftlichen Akteurinnen und Akteuren. Nutzungskonkurrenzen im Raum sind aufgrund von Flächenknappheit vorprogrammiert, sodass die Akzeptanz der erneuerbaren Energien in einigen Regionen schon heute an Grenzen stößt. Gleichwohl muss der Ausbau der erneuerbaren Energien weiter voranschreiten, wenn die Energiewende gelingen soll, sodass die Akzeptanz in der Bevölkerung gleichzeitig zu einem Schlüsselfaktor wird. Systematische, alle gesellschaftlichen Gruppen berücksichtigende, das heißt diversity- und genderorientierte, Analysen sind als Basis für das Ermächtigen (empowerment) aller gesellschaftlichen Kräfte unerlässlich, stehen bisher jedoch noch weitgehend aus.

Raum stellt eine zentrale Integrationsebene in der Vermittlung verschiedener gesellschaftlicher Interessen dar; zugleich spiegeln sich in Raumstrukturen, -mustern und -beziehungen gesellschaftliche Machtverhältnisse und Ungleichheitslagen zwischen den Geschlechtern wider (unter anderem Bauriedl/Schier/Strüver 2010; Wastl-Walter 2010). Die machtkritische Analyse der räumlichen Veränderungen im Transformationsprozess ist damit entscheidend für eine nachhaltige, das heißt intra- und intergenerationell gerechte Umsetzung der Energiewende.

Für die räumlichen Planungen entstehen im Kontext der Transformation der Energiesysteme völlig neue Aufgaben, die weit über das traditionelle Planungsverständnis und das klassische Planungsinstrumentarium hinausweisen. Während sich das Aufgabenfeld der Raum- und Landschaftsplanungen im Energiebereich bisher vornehmlich auf die Sicherung von Standorten für Kraftwerke bzw. Anlagen zur Energieumwandlung und Trassen beschränkt hat, stehen die Planungen heute und zukünftig vor der Herausforderung, den vielfältigen Wandel von der Kulturin die Energielandschaft konstruktiv mitzugestalten. Da es hierbei um weit mehr als um Abwägung von Nutzungsoptionen geht - nämlich um die gesellschaftliche Akzeptanz für neue Raumfunktionen, -typen und -bilder - gewinnen kooperative und partizipative Planungsverständnisse, die Entwicklungs- und Ordnungsfunktionen sowie formale und informelle Instrumente einschließen, weiter an Bedeutung. Als geeignete Ebenen für derartige weitgefasste, kollektive Gestaltungsprozesse der Energiewende gelten - aufgrund der mit der Nutzung erneuerbarer Energien verbundenen
Dezentralisierung des Energiesystems - die lokale und besonders die regionale Ebene oberhalb von Kommunen und unterhalb der Bundesländer (BMVBS 2011; Beckmann/Gailing/Hülz et al. 2013; Gailing/Hüesker/Kern et al. 2013). Eine bedeutende, koordinierende Funktion wird auf der regionalen Ebene der Regionalplanung zugesprochen (BMVBS 2011; BMVBS 2013a; Gailing/Röhring 2015: $37 \mathrm{ff}$.). Jedoch werden für diese in der Praxis auch erhebliche Schwierigkeiten konstatiert, die aus der widersprüchlichen Rolle resultieren, einerseits weiterhin verschiedene Raumbelange zu koordinieren und andererseits der neuen Rolle als „Energieakteur“ (z. B. als federführende Institution bei der Aufstellung regionaler Energiekonzepte) $\mathrm{zu}$ entsprechen (Gailing/Hüesker/Kern et al. 2013: 38). Darüber hinaus wird die Entwicklung der Regionalplanung in Richtung einer proaktiven Raumplanung z. B. auch durch Defizite in der Ausbildung oder fehlende Personalressourcen erschwert (Fürst 2011: 62). Die Landschaftsplanung spielt daneben eine zentrale Rolle für die Sicherung und Weiterentwicklung der ökologischen Funktionen der Landschaften, die einem hohen Transformationsdruck unterliegen (SRU 2008: Tz 233; BfN 2010: 28; BfN/BBSR 2014a; BfN/BBSR 2014b). Die planungswissenschaftliche Forschung und praktische Ausgestaltungen der neuen Planungsaufgaben befinden sich noch am Anfang.

Ziel unseres Beitrags ist es, erste konzeptionelle Bausteine für eine an Nachhaltigkeit und Geschlechtergerechtigkeit orientierte, raum- und planungsbezogene Energieforschung aufzuzeigen. Damit wollen wir die Innovationspotenziale einer auf gesellschaftliche Naturverhältnisse blickenden Geschlechterforschung erschließen, die als „EyeOpener" fungiert: In analytischer Perspektive sollen in Dichotomien (z. B. kulturell - natürlich, urban - ländlich) eingelassene Hierarchisierungen (Auf- und Abwertungen) sichtbar und für die macht- und herrschaftskritische Ungleichheitsforschung nutzbar gemacht werden, um damit die Potenziale für eine substanziell und prozedural nachhaltige Gestaltung der Energiewende zu erweitern. Geschlecht verstehen wir dabei zum einen als eine Analysekategorie, die es ermöglicht, Gerechtigkeits- und Demokratiedefizite sowie herrschaftliche Umgangsweisen mit Natur frühzeitig sichtbar zu machen. Zum anderen lassen sich auf der Grundlage einer solchen geschlechterorientierten, machtund herrschaftskritischen Analyse der in den Transformationsprozessen des Energiesystems neu entstehenden Handlungsräume Potenziale für die Generierung von Transformationswissen für eine emanzipatorische (Um-) Gestaltung der Energiewende im Raum erkennen. Wir skizzieren somit im Folgenden eine Heuristik im Sinne der Transformationsforschung (WBGU 2011: 342 f.), die perspektivisch als transformativer Forschungsansatz in der inter- und transdisziplinären Raumforschung zur Energiewende angewen- 
det werden kann, um neue Denk- und Handlungsräume für eine nachhaltige Raumentwicklung zu öffnen.

Hierzu betrachten wir aus der Geschlechterperspektive zunächst den Stand der raum- und planungsrelevanten Energie- und Klimaforschung (Kapitel 2). Ausgehend von der sozial-ökologischen Forschungsprogrammatik stellen wir in Kapitel 3 vier Analyseperspektiven dar, die sich in der nachhaltigkeitsbezogenen Geschlechterforschung finden und mit einem jeweils spezifischen Verständnis von „Geschlecht“" unterlegt sind. Diese Perspektiven werden in Kapitel 4 exemplarisch auf raum- und umweltbezogene Fragen der Energiewende angewandt, um die Potenziale der Kategorie Geschlecht für die raum- und planungswissenschaftliche Forschung im Handlungsfeld Energie zu eruieren und als (vorläufige) Forschungsperspektiven zur Diskussion zu stellen. Hierauf aufbauend entwickeln wir in Kapitel 5 Ausblick(e) für die weitere räumliche Planung und planungsbezogene Energieforschung.

\section{Zum Stand der Energie- und Klimaforschung aus Geschlechterperspektive}

Das auf fossilen Energieträgern beruhende Erzeugungs- und Versorgungssystem geht mit ökologischen und sozialen Risiken einher - das gilt sowohl in Bezug auf die Klimaveränderungen durch anthropogene Treibhausgasemissionen als auch in Bezug auf die Risiken durch atomare Stromproduktion. Deshalb sind Forschungen zur Nutzung erneuerbarer Energien und zur Transformation der Energiesysteme mit jenen zum Klimawandel und dessen Folgen sowie mit Forschungen zur Transformation in eine postfossile Gesellschaft eng verbunden. Ebenso sind Politiken und Planungen für die Energiewende nicht ablösbar von Klimaschutzzielen; sie sind (mindestens in Deutschland) erst vor dem Hintergrund der politischen Debatten um Klimaschutz und den gesellschaftlichen Umgang mit den Klimafolgen entstanden. Doch bleibt der beide Forschungs- und Politikfelder verbindende Blick auf die hierin zum Ausdruck kommende Krise gesellschaftlicher Naturverhältnisse (Becker/Hummel/Jahn 2011: 82 ff.) und das damit verbundene Potenzial für eine sozial-ökologische Transformation dann unverstanden, wenn die Debatten sich auf technische und ökonomische Fragen konzentrieren, wie dies im deutschsprachigen Raum derzeit noch vorwiegend geschieht (zur Übersicht z. B. BMWi 2011; BMU 2013). Diese Vernachlässigung der gesellschaftlichen Dimension führt auch zu einer systematischen Ausblendung der Bedeutung von Geschlechterverhältnissen (vgl. z. B. Wiliarty 2011). Wir gehen davon aus, dass eine sozial-ökologische Perspektive das Potenzial hätte, die Energie- und Klimaforschung miteinander zu verbinden und in dieser Verbindung zugleich zu einer Erweiterung der Forschungsperspektiven beizutra- gen: Indem Wissen nicht mehr abgelöst von seinen sozial-ökologischen Kontexten erzeugt und als vermeintlich universell gültiges Wissen in Politikkonzepte eingelassen wird, erscheinen die Energiewende und der Klimawandel als Transformationsprozesse, in denen sich die gesellschaftlichen Naturverhältnisse wandeln. Da die Kategorien Natur und Geschlecht untrennbar miteinander verbunden sind (vgl. Schultz/Hummel/Hayn 2006), finden diese Transformationsprozesse ihren Ausdruck auch in gesellschaftlichen Geschlechterverhältnissen, sind also nicht geschlechtsneutral und wirken (natur- und sozial-) räumlich.

Neu gegründete Netzwerke und Plattformen lassen zukünftig Fortschritte in Bezug auf die „,blinden Flecken“ der Energie- und Klimaforschung erwarten, wie das von Frauen aus Forschung und Industrie gegründete Netzwerk „European Network of Women for Innovative Energy Solutions“ (Women4Energy) ${ }^{5}$, das disziplinenübergreifende „Forschungsforum Energiewende" ${ }^{\prime 6}$ und im Hinblick auf urbane Räume und den Klimawandel die „Nationale Plattform Zukunftsstadt ${ }^{\text {“7 }}$. Doch stehen die Forschungen zu gesellschaftlichen Dimensionen der Energiewende insgesamt noch am Anfang. So lassen sich bisher nur vereinzelt Forschungsansätze identifizieren, in denen Geschlechterperspektiven angelegt sind. Ins Blickfeld rückt hier insbesondere der inter- und transdisziplinäre Förderschwerpunkt „Umwelt- und gesellschaftsverträgliche Transformation des Energiesystems" im Rahmen der Sozial-ökologischen Forschung (SÖF) des Bundesministeriums für Bildung und Forschung (BMBF), der 2013 mit ca. 30 Projekten gestartet ist. ${ }^{8}$ Da das Anliegen, neben Systemwissen zugleich auch Transformationswissen zu generieren, explizit Teil dieses Forschungsprogrammes ist, lassen sich ausgehend von diesen Projekten weitreichende Erkenntnisse zu Fragen sozial-ökologischer Transformationen erwarten. Jedoch bleibt abzuwarten, ob und wie geschlechterbezogene Forschungsfragen und -perspektiven in diese Forschungen eingehen werden, da die Betrachtung der „Problemdimension Gender \& Environment“" (Becker/Jahn/Schramm 1999: 43 ff.) nicht mehr prinzipiell im Forschungsprogramm verankert ist (Schultz/Schramm/Hummel 2015: 227). So wird die Dimension „Gender“ bisher nur in einem Projekt explizit genannt (BMBF 2014). Doch lässt sich aufgrund der theoretisch-konzeptionellen Verankerung der SÖF im Konzept gesellschaftliche Naturverhältnisse (vgl. Kapitel 3), in dem die Reflexion der Kategorie Geschlecht bei der Analyse der basalen Ordnungsmuster von Gesellschaften als unver-

\footnotetext{
5 https://www.steinbeis-europa.de/index.php5? mode $=$ main $\&$ file $=663 \&$ dev_id=1279 (18.5.2016).

${ }^{6} \mathrm{http} / / / \mathrm{www}$. acatech.de/forschungsforum-energiewende (18.5.2016).

7 http://www.difu.de/projekte/2013/geschaeftsstelle-nationale-plattformzukunftsstadt-npz.html (06.03.2015).

8 vgl. http://www.transformation-des-energiesystems.de(13.5.2016).
} 
zichtbar gilt (Becker/Hummel/Jahn 2011: 79), erwarten, dass Forschungsperspektiven auf Geschlechterverhältnisse in weiteren Projekten eine Rolle spielen werden (vgl. auch Schultz/Schramm/Hummel 2015: 227). Für die ErschlieBung von Geschlechterperspektiven gilt es daher, dieses neue Forschungsfeld systematisch mit in den Blick zu nehmen.

Schwerpunkte der auf Klima- und Energiefragen bezogenen internationalen Geschlechterforschung liegen bisher vor allem im globalen Süden. Sehr häufig sind sozialökologische Problemlagen in Ländern des globalen Südens direkt und offensichtlich mit den Folgen des Klimawandels verwoben, sodass Ressourcenpolitiken und -planungen Geschlecht und andere Ungleichheitsdimensionen zu integrieren haben. Die Diskussionen hierzu auch und gerade in der Geschlechterforschung - haben daher bereits Tradition und befassen sich insbesondere mit ungleichen Machtverhältnissen von Männern und Frauen sowie geschlechtsspezifischen Arbeitsteilungen in Entwicklungs- und Schwellenländern, häufig in feministischer Perspektive (z.B. Agarwal 1998; Lambrou/Piana 2006; FES 2008; Brownhill/Turner 2009; Hemmati/Röhr 2009; Caglar/Castro Varela/Schwenken 2012; Bauriedl/ Wichterich 2014: 24 ff.; Ryan 2014: 97 ff.).

Im europäischen Kontext liegen Geschlechterforschungen zu klima- und energierelevanten Themen wie Mobilität (z. B. Ahrend/Herget 2013; Bauhardt 2007), Stoffströme und Konsum (Weller 2004; Weller 2012; Weller 2013) seit den 1980er-Jahren in zahlreichen Studien vor. Erst in jüngerer Zeit entwickelt sich ein eigenständiger Forschungsstrang zu Klimawandel, -schutz und -politik aus Geschlechterperspektive (z. B. Hemmati/Röhr 2009; Bauriedl/ Schier/Strüver 2010), der Fragen nach Geschlechtergerechtigkeit im Transformationsprozess der Energiesysteme partiell einschließt (z. B. Bauriedl 2013; Bauriedl/Wichterich 2014: 20 ff.; Fraune 2015). Dies gilt ebenso für Studien, die sich mit Energiethemen aus der geschlechterorientierten Perspektive von Konsumentinnen und Konsumenten befassen (z. B. Röhr 2001; Fischer 2011; Röhr/Alber/Kösters 2012; Hübner/Müller/Röhr o.J.; Weller 2013). Beispielsweise wird in einer im EU-Kontext angelegten Studie des Instituts für sozial-ökologische Forschung (ISOE) (Schultz/ Stieß 2009) das Thema Geschlecht und Energiekonsum ausführlich behandelt: Es werden Unterschiede zwischen Männern und Frauen in Bezug auf den Energieverbrauch im Haushalt, die Entscheidungsmacht über Energieverwendung und Art der genutzten Energie sowie in den Einstellungen zu Energiereduktionen, Risiken durch Energieproduktion und Energietechnologien deutlich. Zugleich zeigt die Studie jedoch auch, dass differenztheoretisch angelegte Erhebungen nur begrenzt aussagefähig sind, wenn die Ergebnisse nicht in Relation zu anderen Differenzfaktoren wie Einkommen, Alter, Größe der Haushalte u. a. betrachtet werden (Clancy/Röhr 2003: 38; Hofmeister/Katz/Mölders 2013a: 37 ff.).

Bisher unverbunden und noch ohne explizite Perspektiven auf Geschlechterverhältnisse beginnen sich Forschungen zu Implikationen der Energiewende im Raum dynamisch zu entwickeln, z. B. in Bezug auf neue Geographien der Energiesysteme (Arbeitskreis Geographische Energieforschung) $)^{9}$, zu regionalen Selbstversorgungsinitiativen und regionalwirtschaftlichen Fragestellungen (z.B. Aretz/Kress/Schlager 2011; Moser 2011; Hirschl/Aretz/ Böther 2011; Hirschl/Salecki/Böther et al. 2011) bis hin zur Entwicklung einer neuen Forschungsheuristik für die raumwissenschaftliche Energieforschung (Moss/Gailing/ Kern et al. 2013). Besonders aus Letzterer versprechen die für den Zeitraum von 2015-2018 anvisierten Analysen neuer Räume der Energiewende im Spannungsfeld von Macht, Materialität und Menschen (IRS o.J.: 20 ff.) einen Fundus für Geschlechterperspektiven zu bieten, den es systematisch mit auszuleuchten gilt.

Raum- und planungswissenschaftliche Forschungsarbeiten mit explizitem Bezug auf die Geschlechterforschung haben sich seit den 1970er- und 1980er-Jahren vor allem in der Geographie und in den Planungswissenschaften etablieren können (z. B. Massey 1994; Becker/Neusel 1997; Bauriedl/Schier/Strüver 2010; Wastl-Walter 2010). Ansätze geschlechterbezogener Raumanalysen und des „GenderPlanning" in der Perspektive auf sozial-ökologische Transformationen beziehen sich insbesondere auf Fragen zur Urbanisierung und Stadtentwicklung (z. B. auch Bauriedl/ Wichterich 2014: 27 ff.), also auf die kommunale Planungsebene. Dabei sind die Übergänge zu einer sozialökologischen Raumforschung auf regionaler Ebene flieBend (z. B. Hofmeister/Scurrell 2006; FBW 2007). Auch die geschlechterbezogene Forschung zu ländlichen Räumen hat eine lange Tradition, war jedoch weitestgehend auf den Agrarsektor beschränkt und setzt sich erst in jüngerer Zeit auch mit Fragen nachhaltiger ländlicher Entwicklung aus Geschlechterperspektive auseinander (vgl. Schmitt 2005). Bislang sind die Ansätze raumwissenschaftlicher Geschlechterforschung jedoch nur selten mit Fragen nach den Ver- und Entsorgungsstrukturen sowie nach der Entwicklung von technischer Infrastruktur verknüpft (wie z. B. Kruse 2010; auch Knothe 2013). Die Entwicklung von geschlechterbezogenen Forschungsansätzen zur Energiewende in einer raumwissenschaftlichen Perspektive steht derzeit noch am Anfang (Kanning 2013).

Noch im Anfangsstadium befindet sich auch die raumbezogene, planungswissenschaftliche Energieforschung insgesamt. Einhergehend mit den dynamischen Entwicklungen der erneuerbaren Energien lässt sich diese noch eher als reaktiv und „work in progress“ (Klagge 2013: 9) cha-

\footnotetext{
${ }^{9}$ http://www.geographische-energieforschung.de/ (18.03.2016).
} 
rakterisieren. Mit fortschreitender Realisierung der neuen, dezentralen Energiesysteme im Raum entwickelt sich diese jedoch zugleich dynamisch weiter. Bezogen sich die Arbeiten bisher vornehmlich noch auf einzelne Energieträger, z. B. Windenergie und Biomasse sowie damit verbundene (natur-)räumliche Auswirkungen und Akzeptanzfragen (zusammenfassend z. B. DLR 2006), befassen sich neuere Arbeiten insgesamt mit dem Wandel der Kultur- zu Energielandschaften und den damit verbundenen Herausforderungen für die Raum- und Landschaftsplanung (z. B. BfN/ BBSR 2011). Aktuelle Forschungen richten sich zudem auf den Netzausbau und konzentrieren sich dabei vornehmlich auf das formale Planungsinstrumentarium (z. B. Hanusch/ Einig/Peters et al. 2012). Darüber hinaus stehen seit Kurzem integrierte Planungsansätze wie regionale Energiekonzepte und Governance-Prozesse im Fokus. Diese weisen unter anderem auf notwendige Weiterentwicklungen der räumlichen Planung im Hinblick auf die erforderliche Verzahnung von informellen und formellen Instrumenten sowie eine (verbesserte) vertikale Koordination hin, um „Nutzen und Lasten“ der erneuerbaren Energien sozial und ökologisch gerecht zu verteilen (z. B. BfN 2010; BfN/BBSR 2011; BMVBS 2011; BMVBS 2013a; BMVBS 2013b; Gailing/Leibenath 2013; Klagge/Arbach/Franck et al. 2013) und bestätigen eigene Forschungsarbeiten (Kanning/Buhr/Steinkraus 2009; Wiehe/Buhr/Wolf et al. 2010). Geschlechterperspektiven spielen bei allen Ansätzen noch keine Rolle.

Um diese weiterzuentwickeln, gälte es, die aus raumwissenschaftlicher Sicht gewonnenen Erkenntnisse der Geschlechterforschung systematisch auf das Transformationsfeld Energie zu beziehen und mit den im Bereich Ressourcenpolitik und -planung generierten Erkenntnissen der Geschlechterforschung zu verbinden. Dies gilt ebenso für Studien, die erste geschlechterdifferenzierte Einblicke in Investitionsentscheidungen von Haushalten in energiesparende Technologien und/oder für erneuerbare Energien betreffen (Hübner/Müller/Röhr o. J.; Schultz/Stieß 2009; Röhr/ Alber/Kösters 2012). Diese in ihren räumlichen Kontexten zu betrachten und sie damit beispielsweise für Fragen zur räumlichen Gestaltung und Effizienzverbesserung der Wärmeversorgung nutzbar zu machen, stellt eine vielversprechende Forschungsaufgabe dar. Denn deutlich würde hiermit, dass und inwieweit die Kopplung der Forschung zum Transformationsfeld Energie an das Alltagswissen der Akteurinnen und Akteure besondere Erkenntnisse zu generieren verspricht.

Die Frage, ob und wie das Alltagswissen der an der Energiewende Beteiligten und von ihr Betroffenen in energieund klimapolitische Entscheidungen eingeht und inwieweit alltägliche Praktiken Potenziale oder Hindernisse für Transformationsprozesse sind, verweist auf das Feld der sich entwickelnden, energiebezogenen Akzeptanz- und Partizipationsforschung (z. B. Schweizer-Ries/Rau/Hildebrand
2011; Zoellner/Rau/Schweitzer-Ries 2011). Hierzu liegen vereinzelt Ansätze aus der Geschlechterforschung vor, die Hinweise auf spezifische Unterschiede und neue Ungleichheiten zwischen den Geschlechtern geben (z. B. von Winterfeld 2012). Für das Transformationsfeld Energie liegen jedoch Partizipationsforschungen kaum (Ausnahme Radtke 2014) und systematisch nach Geschlecht differenzierte Analysen nur vereinzelt (Fraune 2015) vor. Die wenigen vorhandenen Studien greifen raumwissenschaftliche Forschungsergebnisse (z.B. Bauhardt 2004) nicht auf und sind nicht mit planungstheoretischen Ansätzen, wie z. B. „Equity Planning“ (Fainstein/Fainstein 1996), die Teilhabechancen marginalisierter Bevölkerungsgruppen an energiepolitischen Entscheidungen reflektieren, verbunden. Dabei gälte es, Partizipationsforschung standpunktbezogen, das heißt in Abhängigkeit von der jeweiligen gesellschaftlichen Positionierung, zu differenzieren und die Ergebnisse in Empfehlungen zur Weiterentwicklung der Planungsund Steuerungsstrukturen einzubringen. Im Hinblick auf die Governance-Forschung, welche die planungstheoretischen Diskurse weitgehend prägt (vgl. Hofmeister 2014), könnten derartige Forschungen zur Entwicklung eines neuen Planungsverständnisses im Sinne einer strategischen Planung beitragen, eingebettet in eine Mehrebenen-Governance (z. B. Fürst 2011). Denn bislang bleibt auch diese weitgehend geschlechtsneutral, ebenso wie aktuelle raumbezogene Klimawandel-Governance-Forschungen und Empfehlungen (BMVBS 2013a). Dieses gilt übergreifend ebenso für die internationale Transformations- und Transitionsforschung (z. B. Smith/Stirling/Berkhout 2005; Schot/ Geels 2007; Voß/Smith/Grin 2009; Voß/Bornemann 2011), die auch für die Gestaltung der Energiewende als richtungweisend gilt (WBGU 2011). Zwar scheinen kooperative Governance-Formen tendenziell geeigneter, geschlechterkonnotierte Ungleichheiten aufzudecken und aufzulösen, jedoch weisen erste geschlechtsorientierte Aufsätze zur Governance- und Transitionsforschung auch auf fehlende Geschlechter differenzierende Analysen hin (,GenderGap“) (z.B. Brody 2009; Hudson 2012) sowie auf erhebliche Risiken durch die Fortschreibung gewachsener Herrschaftsstrukturen und die Etablierung neuer Ungleichheiten (von Winterfeld 2012; von Winterfeld 2013).

Zusammenfassend bleibt festzustellen, dass die systematische Integration geschlechterorientierter Perspektiven in die Erforschung der sozial-ökologischen und raumbezogenen Fragen zur Energiewende defizitär ist. Jedoch ist einhergehend mit den dynamischen Entwicklungen in der Praxis zunehmende Aufmerksamkeit für gesellschaftliche Fragen, vielleicht gar ein Aufbruch spürbar. Planungsbezogen sind insbesondere substanzielle Bezüge zum Nachhaltigkeitsleitbild offen (Hofmeister 2014) sowie prozedural Fragen der Koordination, sowohl in vertikaler Hinsicht (Mehrebenen-Governance) als auch zwischen informellen 
und formellen Instrumentarien. Geschlechterperspektiven spielen dabei bisher am Rande eine Rolle; dies gilt insbesondere, wie gezeigt, in den Bereichen Klimaschutz, Konsum und Mobilität sowie vereinzelt in der Akzeptanz- und Partizipationsforschung. Wo Geschlechterperspektiven explizit Berücksichtigung finden, das heißt ungleiche Machtverhältnisse zwischen den Geschlechtern offenbart und kritisch reflektiert werden, zeigen sich Potenziale in Hinblick auf neue Problemsichten und -lösungen in nachhaltigkeitsrelevanten Handlungsfeldern mit Bezug auf die Erweiterung durch die Geschlechterperspektive (Hofmeister/Katz/ Mölders 2013b: 339). Aus dieser Perspektive noch völlig unerschlossen sind die neuen Energiesysteme selbst und deren nachhaltige Gestaltung im Raum, z. B. im Hinblick auf Zentralität vs. Dezentralität. Von einer geschlechterorientierten sozial-ökologischen Forschung zur Transformation der Energiesysteme im Raum kann - neben einer stärkeren Anbindung der Wissensgenerierung an Alltagswissen und lebensweltliche Problemlagen (Kontextualisierung des Wissens) - erwartet werden, dass Trennungsverhältnisse und hierarchisierte Dichotomien stärker als bisher in den Fokus kritischer Analysen geraten. Die Entwicklung von Systemwissen zum Transformationsfeld Energie wird so zu einer standpunktorientierten Forschung (z. B. Ryan 2014: 97), von der aus Ziel- und Transformationswissen generiert werden kann. So wird in dieser Perspektive sichtbar, dass Machtverhältnisse sich im Transformationsprozess einerseits gesellschaftlich manifestieren und räumlich wirksam sind. Andererseits lassen sich jedoch auch Verschiebungen und Hybridisierungen in und zwischen den die moderne Gesellschaft prägenden Dichotomien (wie Produktions- vs. Reproduktionssphäre, Produktion vs. Konsumtion, Stadt vs. Land) erkennen; sie begleiten den Transformationsprozess und ermöglichen - neben der Verschiebung bestehender und der Ausbildung neuer Hierarchisierungen - zugleich auch die Ausbildung neuer Machtverhältnisse und Bezogenheiten (auch FBW 2007), die zu mehr Gerechtigkeit führen können. Ob und wie die kritischen Analysen der Geschlechterforschung fruchtbar auf die Forschungs- und Handlungsfelder zur Energiewende angewendet werden können, gilt es systematisch zu erforschen. Dabei werden die analytischen Perspektiven der nachhaltigkeitsorientierten Geschlechterforschung anwendbar. Dies wird im Folgenden exemplarisch dargestellt.

\section{Analytische Perspektiven aus der sozial- ökologischen Nachhaltigkeits- und Geschlechterforschung}

Im Unterschied zum WBGU (2011), der von der Notwendigkeit einer „Großen Transformation“ ausgeht, zeigt sich in sozial-ökologischer Perspektive, dass es vieler und viel- fältiger Transformationsprozesse in sozialer, kultureller, ökonomischer und ökologischer Hinsicht und auf verschiedenen Handlungs- und räumlichen Ebenen bedarf, um den gegenwärtigen Krisenherden - wie dem Klimawandel, dem Verlust von Ökosystemleistungen und biologischer Vielfalt, dem demographischen Wandel und der Erosion der sozialen Reproduktionssysteme - wirksam entgegenzutreten. In diesem Verständnis verorten wir unseren Beitrag im Forschungsprogramm Soziale Ökologie (BMBF 2000), in dem das Konzept gesellschaftlicher Naturverhältnisse die Zentralreferenz darstellt. In diesem als „Orientierungsrahmen" verstandenen Konzept stehen die Beziehungen zwischen Gesellschaft und Natur im Vordergrund, die sowohl unter materiell-physischen als auch unter symbolischkulturellen Aspekten verstanden und in Wechselwirkung zueinander analysiert werden (Becker/Hummel/Jahn 2011: 78). Dabei konzentriert sich die sozial-ökologische Forschung auf basale gesellschaftliche Naturverhältnisse - das sind solche, die sowohl für die gesellschaftliche als auch für die individuelle Reproduktion und Entwicklungsfähigkeit unverzichtbar sind. Beispiele hierfür sind Arbeit und Produktion, Sexualität und Fortpflanzung, Ernährung, Mobilität und Kommunikation (Becker/Hummel/Jahn 2011: 78 f.). Die Orientierung an „Grundbedürfnissen“ als basalen Naturverhältnissen gibt dem Konzept eine normative Orientierung, das heißt, dem Leitbild der Nachhaltigen Entwicklung entsprechend muss deren Regulierung gelingen, um die Reproduktions- und Entwicklungsfähigkeit sowohl der Gesellschaft als auch ihrer natürlichen Lebensgrundlagen zu sichern (BMBF 2000: 6; vgl. auch Becker/Hummel/ Jahn 2011: 79). Dem Begriff der Grundbedürfnisse liegt ein anthropologisches Verständnis zugrunde, das Menschen als Natur- und Kulturwesen bestimmt; Grundbedürfnisse existieren nur in gesellschaftlich interpretierter, kulturell geformter Weise. Materiell-physische und kulturell-symbolische Aspekte sind daher nicht voneinander trennbar (Becker/Hummel/Jahn 2011: 79). Indem im Konzept gesellschaftlicher Naturverhältnisse Grundbedürfnisse als zentrale Kategorie verankert sind und die normative Orientierung am Leitbild Nachhaltiger Entwicklung begründen, schließt das Konzept in seinen normativen Grundlagen an raum- und planungswissenschaftliche Debatten zur Sicherung der „Daseinsgrundfunktionen“ (Partzsch 1970) als Grundlage einer nachhaltigen Raumentwicklung an.

In der Analyse gesellschaftlicher Naturverhältnisse ist die Reflexion der Kategorie Geschlecht insofern unverzichtbar, als ,gesellschaftliche Naturverhältnisse als Geschlechterverhältnisse strukturiert [sind] und diese [strukturieren]. Geschlecht bzw. Geschlechterdifferenz stellt ein basales Ordnungsmuster von Gesellschaft dar" (Becker/ Hummel/Jahn 2011: 79). Geschlechterverhältnisse bilden daher für die sozial-ökologische Forschung eine zentrale 


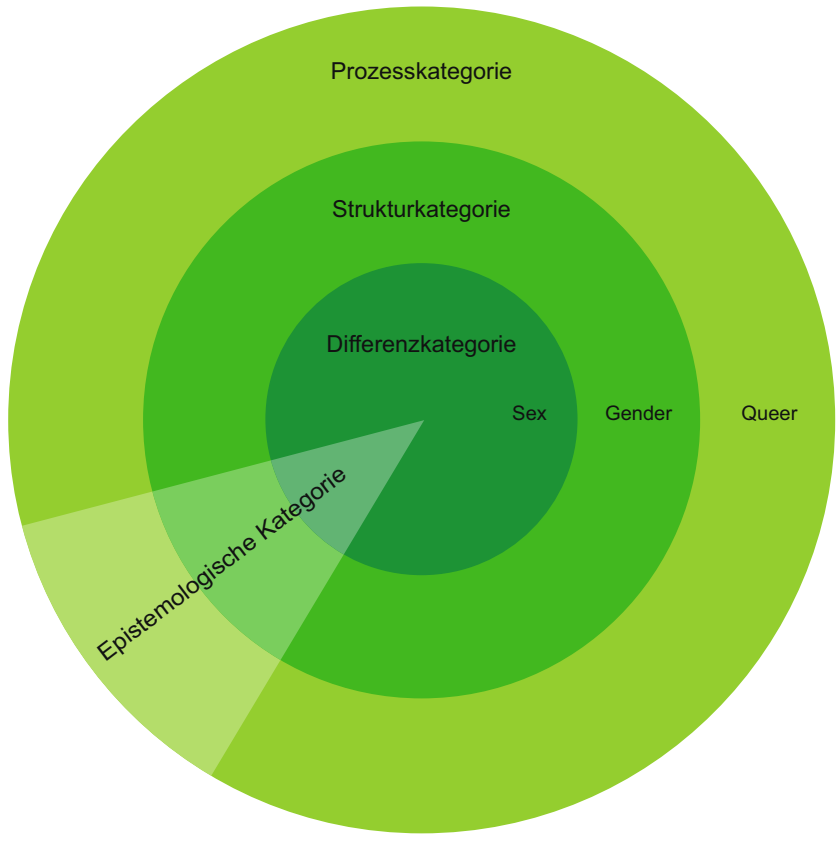

Abb. 1: Vier analytische Perspektiven der geschlechterorientierten Nachhaltigkeitsforschung (nach Hofmeister/Katz/Mölders 2013a: 47 ff.)

Problemdimension (BMBF 2000: 19)..$^{10}$ Ebenso wie gesellschaftliche Naturverhältnisse insgesamt werden auch Geschlechterverhältnisse im Sinne eines pluralen Verständnisses der Beziehungen zwischen den Geschlechtern verstanden (Becker/Hummel/Jahn 2011: 79). Für das Begreifen der Beziehungsmuster - z. B. zwischen Kultur und Natur, männlich und weiblich - ist es im Forschungsprogramm Soziale Ökologie unerlässlich, Dualismen in kritischer Absicht aufzudecken mit dem Ziel, sie als solche zu überwinden. Denn von einem dualistischen Standpunkt aus ,sind nur noch zwei sich gegenseitig ausschließende Bestimmungen der Beziehungen möglich: Naturalisierung der Gesellschaft oder Vergesellschaftung der Natur“ (Becker/Hummel/Jahn 2011: 88 im Rekurs auf Eder 1988: 27). Ausgehend von der Intention, sowohl naturalistische als auch kulturalistische Reduktionen in der Bestimmung gesellschaftlicher Naturverhältnisse zu vermeiden und stattdessen einen vermittlungstheoretischen Weg in der Analyse der Beziehungsmuster zu entwickeln (Kropp 2002; vgl. Fußnote 4), wird die Unterscheidung zwischen Natur und Gesellschaft nicht als eine ontologische, sondern als eine sozial konstruierte, historisch besondere Unterscheidung theoretisiert - eine polarisierende Unterscheidung, mit der

\footnotetext{
10 Zur analytischen Erklärung der wechselseitigen Strukturierung von Natur- und Geschlechterverhältnissen existieren - neben der Theoretisierung dieser Verknüpfung im Konzept gesellschaftliche Naturverhältnisse als Orientierungsrahmen der sozial-ökologischen Forschung (vgl. hierzu Hofmeister/Mölders 2006) - weitere Ansätze (für eine Übersicht vgl. Hofmeister/Katz 2011).
}

jeweils spezifische Bewertungen bzw. Hierarchisierungen verknüpft sind (Becker/Hummel/Jahn 2011: 89).

An dieser Stelle verbindet sich die Heuristik sozial-ökologischer Forschung mit jenen Heuristiken, die für die Geschlechterforschung typisch sind: Auch hier geht es um die Bestimmung von Geschlechterdifferenzen in sowohl materieller als auch in symbolischer Dimension sowie darum, die den männlich und weiblich konnotierten Zuordnungen und Dualismen innewohnenden Hierarchisierungen in kritischer Absicht aufzudecken. Auch die Diskurse um „Geschlecht" können als in naturalistische und kulturalistische Lager gespaltene angesehen werden. Dabei werden immer wieder Ansätze zur Überwindung dieser Spaltung - zwischen den sich ausschließenden Prämissen „Geschlecht als Natur" oder „Geschlecht als soziales Konstrukt" vermittelnd - in die Diskussion eingebracht (vgl. hierzu unter anderem Kropp 2002; Alaimo/Hekman 2008; Hofmeister/ Katz/Mölders 2013a: 33 ff.; Holland-Cunz 2014).

Vor diesem Hintergrund und mit der Intention, solche vermittlungstheoretischen Ansätze für Forschungen im Überschneidungsbereich von Nachhaltigkeits-, Geschlechter- und Raumforschung fruchtbar zu machen, unterscheiden wir im Folgenden vier verschiedene analytische Zugänge zur Kategorie Geschlecht, um diese für die raum- und nachhaltigkeitsorientierte Erforschung der sozial-ökologischen Transformation im Handlungsfeld Energie fruchtbar zu machen. Je nach Fragestellung, Untersuchungsgegenstand und Gestaltungsanspruch erscheinen diese unterschiedlichen analytischen Zugänge sinnvoll. Für die Transformationsforschung lassen sich - in Anlehnung an Hofmeister/Katz (2011) und Hofmeister/Katz/Mölders (2013a: 47 ff.) - vier Analyseperspektiven unterscheiden, die mit jeweils spezifischen Verständnissen der Kategorie Geschlecht unterlegt sind und sich für das Transformationsfeld Energie als weiterführend erweisen (vgl. Abb. 1):

Analytische Zugänge, die von Geschlecht als Differenzkategorie ausgehen, fragen nach Unterschieden in den $\mathrm{Zu}-$ ständigkeiten, Verwundbarkeiten und Betroffenheiten im Bewusstsein und in Wahrnehmung und Verhalten von (biologischen) Männern und (biologischen) Frauen (Hofmeister/Katz/Mölders 2013a: 47, 50 ff.). Sie stellen den theoriegeschichtlichen Ausgangspunkt einer frauenpolitisch motivierten Forschung dar und sind ,verbunden mit dem (politischen) Anliegen der Frauen- und Geschlechterforschung, Ungleichheit und Diskriminierung von Frauen sichtbar und kritisierbar zu machen“ (Hofmeister/Katz/Mölders 2013a: 47). Indem von einer Unterscheidbarkeit und von Unterschieden zwischen biologischen Frauen und Männern (Sex) ausgegangen wird, lässt sich die Kategorie Geschlecht empirisch fassen und z.B. in Form geschlechtsspezifisch disaggregierter Erhebungen abbilden. Solcherart „Situationsanalysen" (Weller 2004) werden im Forschungsfeld Umwelt und Nachhaltigkeit genutzt, um z. B. Erkenntnisse da- 
rüber zu gewinnen, wie sich (biologische) Frauen von (biologischen) Männern in ihren Lebensstilen, Konsumverhalten, Umwelt- und Risikobewusstsein und -verhalten unterscheiden. Sichtbar wird, wer - Männer oder Frauen - sich in welchen Tätigkeitsfeldern mit welcher Motivation in welchem Umfang engagiert und/oder diese repräsentiert (Hofmeister/Katz/Mölders 2013a: 50 ff.). Deutlich wird jedoch auch, dass solche differenztheoretisch basierten Erhebungen zu Fehlschlüssen führen können, weil sie oftmals losgelöst von den spezifischen Lebenskontexten bleiben (Hanson 2010: 8, 11). So ist z. B. „Leben mit Kindern“ ein wesentlich deutlicherer Indikator für ein bestimmtes Mobilitätsverhalten als ,Frausein “ - wenngleich auch starke Überschneidungen zwischen beiden Zuordnungen auftreten (vgl. Ahrend/Herget 2013).

Die differenztheoretische Erkenntnisebene wird daher in der Perspektive Geschlecht als Strukturkategorie erweitert (Hofmeister/Katz/Mölders 2013a: 47 f., 62 ff.). Im Fokus stehen hier Untersuchungen von gesellschaftlich-strukturellen Bedingungen, „die Abwertungen und Diskriminierungen und Ungleichheitslagen produzieren und reproduzieren“ (Hofmeister/Katz/Mölders 2013a: 47). Damit verlagert sich die Analyseperspektive von der individuellen auf die gesellschaftliche Ebene. Geschlecht wird als soziale Kategorie begriffen (Gender), und es wird nach den gesellschaftlichen Bedingungsgefügen und Machtverhältnissen gefragt, „die individuelle Handlungsspielräume rahmen und hervorbringen“ (Hofmeister/Katz/Mölders 2013a: 48). Geschlecht wirkt dabei als eine wesentliche gesellschaftliche Strukturkategorie, die sich mit anderen Ungleichheitskategorien wie Klasse, „Rasse“ oder Alter zu „Achsen der Ungleichheit" verbindet (Klinger/Knapp/Sauer 2007). Im Hinblick auf nachhaltigkeitswissenschaftliche Forschungsfelder, wie z. B. Klimawandel oder Energiewende, wäre etwa zu fragen, inwiefern hier neue Tätigkeitsbereiche entstehen, in denen die Kategorie Geschlecht als „Platzanweiserin“ dient, indem bestimmte Tätigkeiten als „reproduktiv“ und damit als sozial „weiblich“ abgewertet werden.

Eine Analyseperspektive, die von Geschlecht als Prozesskategorie ausgeht, stellt eine weitere, theoriegeschichtlich begründete Verlagerung im Verständnis der Kategorie Geschlecht dar (Hofmeister/Katz/Mölders 2013a: 48 f., 67 ff.). Unter dem Einfluss sozialkonstruktivistischer Ansätze wird das Konzept der Zweigeschlechtlichkeit sowohl in seiner biologischen als auch sozialen Dimension infrage gestellt. In der Annahme eines „Doing Gender“ (West/ Zimmermann 1987) wird danach gefragt, wie diese Zweigeschlechtlichkeit durch interaktive Prozesse immer wieder neu hergestellt wird. Damit werden die Kategorien Sex und Gender um eine weitere Kategorie ergänzt: „Queer“ steht für das Nicht-eindeutig-Zuordenbare - bezeichnet Dinge, Handlungen und Personen, die von der Norm der Zweigeschlechtlichkeit abweichen. In Bezug auf nachhaltigkeits- und umweltwissenschaftliche Fragestellungen wird die prozessbezogene Perspektive auf Geschlecht („Doing Gender") insbesondere im Zusammenwirken mit jener auf Natur (,Doing Nature“) fruchtbar (Hofmeister/Katz/Mölders 2013a: 71 f.; vgl. auch Poferl 2001). So wird für ausgewählte Themenfelder wie die Forst- oder Landwirtschaft danach gefragt, wie naturschutz- und nutzungsbezogene Praktiken an der Herstellung von Natur- und Geschlechterverhältnissen beteiligt sind (Katz/Mölders 2013: 274 f.) - eine Forschungsperspektive, die womöglich auch und gerade die raumwissenschaftlichen Debatten zum Transformationsfeld Energie bereichern könnte (vgl. Kapitel 4).

Schließlich ist für die geschlechterorientierte Umweltund Nachhaltigkeitsforschung die Analyseperspektive Geschlecht als epistemologische Kategorie bedeutsam (Hofmeister/Katz/Mölders 2013a: 49, 55 ff.). Diese liegt quer zu den drei oben genannten Zugängen und liefert in Bezugnahme auf die feministische Naturwissenschafts- und Technikkritik „Erkenntnisse über die Prämissen, Theorien, Methoden und schließlich über die Ergebnisse von Wissenschaften" (Hofmeister/Katz/Mölders 2013a: 49). Dabei kann die differenztheoretische Frage nach Frauen in Wissenschaft und Technik ebenso von Interesse sein wie die Frage nach der prozesshaften Herstellung dichotomer Konstruktionen (Subjekt vs. Objekt, rational vs. emotional etc.). Da natur- und ingenieurwissenschaftliche Forschung in nachhaltigkeits- und umweltwissenschaftlichen Feldern von großer Bedeutung ist, leisten die wissenschafts- und technikkritischen Beiträge aus der Geschlechterforschung hier wesentliche Beiträge zu einer sozialwissenschaftlichen Erweiterung der Debatten. Dazu könnte insbesondere an die Arbeiten zu Klimawandel und -schutz von Alaimo (2009) angeschlossen werden.

Es bleibt festzuhalten, dass das Beschreiben, Bewerten und Gestalten von Transformationsprozessen entlang der Analysekategorie Geschlecht facettenreich ist. Erste wissenschaftliche Studien sowie gesellschaftliche Debatten verbleiben bislang jedoch auf der Ebene von Geschlecht als Differenzkategorie. Damit bleiben Impulse, die die Geschlechterforschung sowohl in kritischer als auch visionärer Absicht für die Transformationsforschung im Allgemeinen und zur Forschung im Transformationsfeld Energie im Besonderen leisten könnte, weitgehend unberücksichtigt.

\section{Potenziale geschlechterbezogener Analyseper- spektiven für eine sozial-ökologische Forschung zur Gestaltung der Energiewende im Raum}

Ausgehend von der Erwartung, dass Geschlechterperspektiven für das Transformationsfeld Energie im Raum sowohl für die kritische Analyse als auch für die Entdeckung neuer Denk- und Handlungsräume fruchtbar werden könnten, 
bieten sich die vier eingeführten Analyseperspektiven auf der Basis der Kategorie Geschlecht an.

Die differenztheoretische Analyse von Geschlechterbeziehungen und -verhältnissen ist, wie in Kapitel 3 dargestellt, in der Forschung bereits relativ weitgehend etabliert. Die Ergebnisse sind zum großen Teil auf das Transformationsfeld Energie und Raum anwendbar und lassen sich hierfür weiterentwickeln. So werden z. B. vorliegende Erhebungen zu den nach Art und Umfang unterschiedlichen Verbräuchen und Nutzungen von Energie in Haushalten (Schultz/Stieß 2009: 37 ff.) direkt nutzbar, wenn sie in die Planung und Gestaltung von Verteilnetzen in Siedlungsräumen oder auch in die Weiterentwicklung von „Demand Response“-Strategien (Strategie zur Reaktion auf schwankende Anforderungen) und Energiedienstleistungen auf Stadt- und Quartiersebene eingehen. In einer differenztheoretischen Perspektive kann beispielsweise auch untersucht werden, ob und wie (biologische) Frauen und (biologische) Männer in neuen, durch die Energiewende entstehenden Tätigkeitsfeldern repräsentiert sind. Fragen nach geschlechtsspezifisch unterschiedlichen Wahrnehmungen zu den Auswirkungen der Energiewende (z. B. Landschaftsbildveränderungen) und/oder zu den von neuen Technologien ausgehenden Risiken stellen sich als grundlegende Fragen für die Partizipations- und Akzeptanzforschung im Kontext der Energiewende.

Doch nicht nur auf Betroffenenseite, sondern auch hinsichtlich der Machtverhältnisse in den Akteursgruppen und bei den Governance-Prozessen zur Gestaltung der Energiewende sind geschlechterdifferenzierte Betrachtungen von wesentlicher Bedeutung. Auch dieser auf die Repräsentation von Frauen und Männern in naturwissenschaftlich-technisch dominierten Wissens- und Politikfeldern gerichtete Forschungstyp hat bereits eine recht lange Tradition (vgl. z. B. Ryan 2014: 101 f.), wird jedoch noch zu selten auf Fragen der geschlechtergerechten Gestaltung der Energiewende angewendet. Denn um z. B. besser zu verstehen, welche Gerechtigkeitsaspekte mit der - durch Erzeugung und Einsatz erneuerbarer Energien entstehenden - größeren Nähe von Produktions-/Versorgungssystemen und Verbraucherinnen und Verbrauchern/Konsumentinnen und Konsumenten (,Prosumerinnen und Prosumer") verbunden sind, gilt es, Geschlechterverhältnisse in differenztheoretischer Weise zu untersuchen. Dazu finden sich erste regionale Studien, wie beispielsweise das Projekt „Geschlechtsspezifische Auswirkungen der Energiewende in Südwestfalen“ (Fraune 2015), in dem Daten zu Bürgerbeteiligung und Investitionssummen in Windkraft- und Fotovoltaikanlagen sowie zu Führungspositionen und Teilhabe in politischen Gremien geschlechterdifferenziert erhoben wurden.

Im Hinblick auf das Forschungsfeld ist jedoch bereits deutlich geworden, dass differenztheoretisch basierte Erhebungen um weitere Merkmale, wie z. B. sozialer Sta- tus, Alter, Ethnie, zu erweitern sind (,diversity“), wenn hieraus kontextbezogen handlungsrelevante Aussagen abgeleitet werden sollen (vgl. auch Hofmeister/Katz/Mölders 2013a: 51 f.). Dies gilt auch für die Ergänzung differenztheoretisch basierter Untersuchungen durch solche zu den strukturell wirkenden Geschlechterverhältnissen, das heißt durch Forschungen, die Geschlechterverhältnisse als Vergesellschaftungsprozesse betrachten, die durch die verschiedenen Bereiche der Gesellschaft hindurch machtvoll wirksam werden (Hofmeister/Katz/Mölders 2013a: 62 ff.). Angesichts des Transformationsfeldes Energie wäre etwa zu fragen, inwiefern hier neue Tätigkeitsbereiche entstehen, in denen die Kategorie Geschlecht macht- und herrschaftsförmig wirkt, indem bestimmte Tätigkeiten auf- oder abgewertet werden. Die strukturelle Analyse vermag daher ergänzend zur differenztheoretischen Perspektive sozial-ökologische Konsequenzen der Energiewende aufzuzeigen, die bislang wenig Berücksichtigung finden: Welche Verschiebungen im Trennungsverhältnis Produktion vs. Reproduktion werden gesellschaftlich und räumlich sichtbar, wenn beispielsweise, weiblich ' konnotierte Bereiche (Privathaushalte) die Rolle von (Energie-) Produzentinnen und Produzenten annehmen? Welche Machtverhältnisse stellen sich dabei ein? Wie entfalten diese Wirksamkeit im Raum, z. B. als Trennung zwischen öffentlichen und privaten Räumen - und dies in physisch-materieller und in symbolisch-diskursiver Hinsicht? Und schließlich: Wie sind diese neuen Machtverteilungen unter Gerechtigkeitsaspekten zu bewerten?

Drittens könnte eine prozessbezogene Analyseperspekti$v e$ auf das Transformationsfeld Energie anzulegen z. B. bedeuten, danach zu fragen, inwiefern in Transformationsprozessen bestimmte geschlechterstereotype Dualismen und Hierarchisierungen immer wieder neu hergestellt werden, oder ob sich das Hybride als Kategorie des ,Dazwischens“ durchzusetzen vermag und damit Verschiebungen in und zwischen voneinander getrennten gesellschaftlichen Sphären induziert werden. So kann unter prozessorientierten Aspekten die Geschlechterperspektive beispielsweise auf die Frage nach den Auswirkungen von dezentralen Verteilungsnetzen im Raum gerichtet werden: Welche (neuen) Verbindungen zwischen Versorgerinnen und Versorgern sowie Verbraucherinnen und Verbrauchern (zwischen Produktion und Konsumtion/Reproduktion) werden durch technische Systeme geschaffen? Wie werden Ausgleiche zwischen dem (infolge schwankender Energieeinspeisung bei erneuerbaren Energien) diskontinuierlichen Angebot und der ebenso schwankenden, in der Zeit differenzierten Nachfrage nach Energie (Strom, Wärme) geschaffen, die ausreichend flexibel sind? Die in diesem Zusammenhang anvisierte Strategie einer Bedarfsreaktion (,Demand Response“) geht von einer direkten Koppelung der Energieversorgung an die Funktionen bei den Nachfragerinnen und Nachfra- 
gern und in den Haushalten aus; es wird eine direkte Reaktion der Verbraucherinnen und Verbraucher auf schwankende Angebote angenommen (z. B. Erlinghagen 2014). Die zum Einsatz kommenden neuen Technologien (z. B. mit sogenannten „Smart Meters“ - ,intelligenten“ Zählern - ausgestattete „Smart Homes“) führen zu Verschiebungen innerhalb der und zwischen den geschlechterhierarchisch getrennten Sphären, wie sie räumlich wirksam und planerisch festgelegt sind, z. B. in der Trennung zwischen Wohngebieten, in denen „Reproduktionsarbeit“ geleistet wird, und Gewerbe-/Industriegebieten, in denen sich Erwerbsarbeit realisiert: Gesellschaftlich auszuhandeln sein wird, welche Prozesse und Tätigkeiten innerhalb der sog. Reproduktionssphäre als ,produktive“ zu behandeln wären - und umgekehrt: welche energieverbrauchenden Prozesse in der sogenannten Produktionssphäre, in marktförmigen/erwerblichen Bereichen als ,konsumtiv' gelten können. So wird z. B. die Frage, ob und wie energieverbrauchende, reproduktive“ Tätigkeiten, wie Wäschewaschen oder Kochen, im Verhältnis $\mathrm{zu}$ energieverbrauchenden Prozessen in sog. produktiven Bereichen, wie Schaufensterbeleuchtungen und Leuchtreklame, bei knapper Angebotslage zu bewerten sind, offen verhandelt werden müssen (vgl. z. B. Giris-Musy 2014: $130 \mathrm{f}$.).

Indem also andere materiell-physische Prozesse und neue technische Systeme auf gesellschaftliche Prozesse einwirken („Doing Nature“), verändern sich gesellschaftliche Natur- und Geschlechterverhältnisse (,Doing Gender"). In diesen - durch das Porös-Werden der bisherigen Trennungsstrukturen gekennzeichneten - Transformationsprozessen könnten einerseits Hemmnisse für strukturelle und technische Innovationen in der Energiewende liegen: Zum Beispiel könnten die neu entstehenden Koppelungen zwischen Produktions-/Versorgungs- und Konsumtions-/ Verbrauchssystemen in der Zeit (,Doing Nature“) neue Geschlechterhierarchien dann erzeugen, wenn die Energieversorgungssysteme einen direkten restriktiven Einfluss auf die (Re)Produktionsarbeitsstrukturen erhielten („,Doing Gender"). Andererseits werden aber auch Chancen für eine sozial-ökologische Transformation mit emanzipatorischen Potenzialen deutlich: Dass und wie weit nicht marktförmige Prozesse und nicht erwerbliche Tätigkeiten als produktive - und nicht, wie bisher, als konsumtive - zu bewerten sind, und umgekehrt, dass und wie weit in die Marktsphäre eingebundene Prozesse konsumtiven Charakter haben, wird gesellschaftlich auszuhandeln sein. Die Verständnisse von „Arbeiten“ und „Wirtschaften“ und die hierin eingeschriebenen geschlechterhierarchischen Bewertungen werden fragwürdig: Die tradierten Geschlechterverhältnisse brechen auf und beginnen sich in solchen Prozessen neu auszurichten. Doch wer hat dabei die Definitionsmacht? Wer entscheidet über das Verhältnis von Angebotsund Nachfragerechten und -strukturen? Und schließlich:
Wie werden räumliche Strukturen und Beziehungen in und zwischen Räumen, z. B. Stadt-Land-Verhältnisse und Stadtstrukturen, von der Etablierung solcher neuen Versorgungsund Nachfragesysteme beeinflusst? Welche Räume werden dabei auf-, welche abgewertet?

Als epistemologische Kategorie wird Geschlecht in der Anwendung auf strukturelle und technische Verursachungen und Wirkungen der Transformation fruchtbar: Werden die Kernthemen der Transformation (gesellschaftliche Transformationspotenziale) ausgeblendet, wenn der Diskurs zur Energiewende vorrangig auf technische Fragen zielt und in den Technikdebatten wiederum auf ökonomische Probleme fokussiert wird? Welche (alten und neuen) Machtverhältnisse sind in die soziotechnischen Systeme der Energieerzeugung, -verteilung und -verwendung eingelassen? Im Transformationsprozess manifestieren sich raumstrukturelle Veränderungen, z. B. durch Bedeutungszuwachs der relativ dezentraleren technischen Versorgungssysteme in der postfossilen Gesellschaft (Beckmann/Gailing/Hülz et al. 2013: 3). Wie sind diese unter (Geschlechter-) Gerechtigkeitsaspekten zu bewerten? Welche neuen Machtverhältnisse und -strukturen verschaffen sich darin Geltung? Welche Funktionen erhalten technische und ökonomische Wissenselemente und Wissensformen im Prozess der Transformation? Und welche Machtverhältnisse werden damit stabilisiert oder erzeugt? Wo immer die Energiewende als ein technisches Transformationsfeld in den Vordergrund der Diskussion rückt, könnte dieser epistemologische Zugang über die Kategorie Geschlecht einen entscheidenden Beitrag zu einer um sozialwissenschaftliche und machtkritische Aspekte erweiterten Debatte leisten (auch Bauriedl 2015: 103).

\section{Ausblick(e)}

Die Energiewende stellt einen sozial-ökologischen Transformationsprozess dar, dessen räumliche - und damit immer auch planerische - Dimensionen noch nicht hinreichend analysiert und verstanden sind. Ein Zugang über die Kategorie Geschlecht verspricht Erkenntnisse aus machtund herrschaftskritischen Blickrichtungen. Damit werden sowohl neue Bestände an Systemwissen als auch Orientierungs-, Ziel- und Transformationswissen generiert. Dies gilt für alle vier vorgestellten, aus der Nachhaltigkeits- und Geschlechterforschung abgeleiteten Analyseperspektiven, für Geschlecht als Differenz-, als Struktur-, als Prozess- wie auch als epistemologische Kategorie. Die kritische Perspektive, die durch die Geschlechterforschung auf Dichotomien und darin eingeschriebene Hierarchisierungen gelegt wird, führt dazu, Gewissheiten in Frage zu stellen und neue Beziehungen zwischen getrennten Sphären zu entwickeln. Die Erforschung des Transformationsfeldes Energiewende 
aus Geschlechterperspektive eröffnet neue Sichtweisen für die räumliche Planung und planungswissenschaftliche Forschung, wie beispielsweise auf folgende aktuell bedeutsame Themenfelder:

\subsection{Von Governance und Partizipation zu Gestaltungsmacht und Teilhabe}

Noch sind die Potenziale der Geschlechterforschung in der - die planungswissenschaftliche Diskussion prägenden prozeduralen Governance- und Partizipations-Forschung weitgehend unerschlossen. Dabei hat die feministische Auseinandersetzung mit dem Politikfeld Nachhaltige Entwicklung deutlich gemacht, dass eine Integration von Geschlechterperspektiven sich nicht in einer (quantitativen) Beteiligung von Frauen an Mainstream-Diskursen erschöpft (Wichterich 2002; Wichterich 2015). Vielmehr geht es darum, marginalisierte Ansätze sichtbar zu machen, in die Diskurse zu integrieren und sie darüber (qualitativ) zu verändern. Es geht um Empowerment und darum, die Gegenstände der Verhandlung mitzubestimmen (von Winterfeld 2013). Dies gilt auch für das Transformationsfeld Energiewende, das sich aus einer Geschlechterperspektive - z.B. als Transformationsfeld von Arbeitsverhältnissen - noch mal anders darstellen mag, als bislang diskutiert. Gestaltungsmacht und Teilhabe aller gesellschaftlichen Kräfte können so auch zu substanziellen Veränderungen im Hinblick auf das Transformationsfeld führen.

\subsection{Zentralität und Dezentralität von Energiesystemen im Raum}

Tendenziell führt die Energiewende mit dem Ausbau der erneuerbaren Energien zu einer Dezentralisierung der Energiesysteme, weil erneuerbare Energieträger wie Sonne, Wind und Biomasse ubiquitär verfügbar sind (vgl. Gailing/ Hüesker/Kern et al. 2013: 41). Jedoch ist die Energieproduktion an die physisch-materiellen Gegebenheiten gekoppelt, die regional sehr unterschiedlich sind. Auch ist die nationale Energiepolitik ebenso wie insbesondere die netzgebundene Stromversorgung eher zentral ausgerichtet (vgl. Gailing/Röhring 2015). Offen ist die Frage, inwiefern dezentrale und/oder zentrale Energiesysteme zu einer nachhaltigeren Gestaltung der Energiewende beitragen. Hierzu könnte beispielsweise die in der Geschlechterforschung entwickelte Vermittlungskategorie der ,,(Re)Produktivität“ (Biesecker/Hofmeister 2006) neue substanzielle Erklärungsbeiträge liefern und damit auch den konzeptionellen Rahmen der räumlichen Planung von der Fläche auf die physisch-materiellen bzw. stofflich-energetischen Prozesse erweitern helfen, worauf auch frühere Arbeiten in vergleichbaren Kontexten hingewiesen haben (Hofmeister 1989; Kanning 2005).

\subsection{Stadt-Land-Beziehungen und die Hybridität von Räumen}

Schließlich sind Geschlechterperspektiven auch für konkret raumrelevante Fragen bedeutsam. Indem auf Beziehungen statt auf Dichotomien geblickt wird, erscheint die Hybridität von Räumen als Qualität. Dies gilt insbesondere hinsichtlich des Stadt-Land-Verhältnisses (Hofmeister/Scurrell 2016). Der Umstand, dass mit der Energiewende die Verhältnisse von Produzentinnen und Produzenten sowie Konsumentinnen und Konsumenten als in städtische und ländliche Räume eingeschriebene Positionierungen neu verhandelt werden, ermöglicht hier Um- und Neubewertungen.

Schon diese kursorischen Annäherungen zeigen: Das Transformationsfeld Energiewende geschlechterorientiert zu erforschen, bedeutet weitaus mehr, als nach „Frauen“ und Energie zu fragen. Es eröffnet sich ein komplexes Forschungsfeld, das die gesellschaftliche Relevanz von vermeintlich technischen Entwicklungen und Innovationen offenbart. Solcherart Offenbarungen sind häufig unbequem, sie irritieren und liegen quer zu unhinterfragten Entwicklungszielen wie Wachstum oder Effizienzsteigerung. Wenn die Energiewende jedoch den Anspruch hat, zu einer nachhaltigen Entwicklung beizutragen, wird sie sich diesen Erweiterungen der Perspektive zu stellen haben und damit auch Räume nachhaltig verändern.

\section{Literatur}

Agarwal, B. (1998): The Gender and Environment Debate. In: Keil, R.; Bell, D. V. J.; Penz, P.; Fawcett, L. (Hrsg.): Political Ecology. Global and Local. London, New York, 193-219.

Ahrend, C.; Herget, M. (2013): Verkehrs- und Mobilitätsforschung aus der Genderperspektive. In: Hofmeister, S.; Katz, C.; Mölders, T. (Hrsg.): Geschlechterverhältnisse und Nachhaltigkeit. Die Kategorie Geschlecht in den Nachhaltigkeitswissenschaften. Berlin, Toronto, 218-227.

Alaimo, S. (2009): Insurgent Vulnerability and the Carbon Footprint of Gender. In: Women, Gender and Research 3-4, 22-35 (= special issue "Gendering Climate Change").

Alaimo, S.; Hekman, S. (Hrsg.) (2008): Material Feminism. Bloomington.

Aretz, A.; Kress, M.; Schlager, P. (2011): Einführung in das Schwerpunktthema - EE-Regionen - Regionale Selbstversorgung mit erneuerbaren Energien. In: Ökologisches Wirtschaften 26, 3, 14-15.

Bauhardt, C. (2004): Entgrenzte Räume. Zu Theorie und Politik räumlicher Planung. Wiesbaden.

Bauhardt, C. (2007): Feministische Verkehrs- und Raumplanung. In: Schöller, O.; Canzler, W.; Knie, A. (Hrsg.): Handbuch Verkehrspolitik. Wiesbaden, 301-319.

Bauriedl, S. (2013): Geschlechterperspektiven auf Klimawandel und -politik. In: Hofmeister, S.; Katz, C.; Mölders, T. (Hrsg.): Geschlechterverhältnisse und Nachhaltigkeit. Die Kategorie Geschlecht in den Nachhaltigkeitswissenschaften. Berlin, Toronto, 235-244.

Bauriedl, S. (2015): Geschlechterdiskussionen in Umweltdiskursen Verfestigung und Aufweichen von Geschlechterdualismen. In: Katz, C.; Heilmann, S.; Thiem, A.; Koch, L. M.; Moths, K.; Hofmeister, S. (Hrsg.): Nachhaltigkeit anders denken. Verän- 
derungspotenziale durch Geschlechterperspektiven. Wiesbaden, $101-113$

Bauriedl, S.; Schier, M.; Strüver, A. (2010): Räume sind nicht geschlechtsneutral: Perspektiven einer geographischen Geschlechterforschung. In: Bauriedl, S.; Schier, M.; Strüver, A. (Hrsg.): Geschlechterverhältnisse, Raumstrukturen, Ortsbeziehungen, Erkundungen von Vielfalt und Differenz im spatial turn. Münster, $10-25$.

Bauriedl, S.; Wichterich, C. (2014): Ökonomisierung von Natur, Raum, Körper. Feministische Perspektiven auf sozial-ökologische Transformationen. Berlin. = Analysen der Rosa Luxemburg Stiftung.

Becker, E.; Hummel, D.; Jahn, T. (2011): Gesellschaftliche Naturverhältnisse als Rahmenkonzept. In: Groß, M. (Hrsg.): Handbuch Umweltsoziologie. Wiesbaden, 75-96.

Becker, E.; Jahn, T.; Schramm, E. (1999): Sozial-ökologische Forschung - Rahmenkonzept für einen neuen Förderschwerpunkt. Frankfurt am Main.

Becker, R.; Neusel, A. (1997): Architektur, räumliche Planung. In: Niedersächsisches Ministerium für Wissenschaft und Kultur (Hrsg.): Berichte aus der Frauenforschung: Perspektiven für Naturwissenschaften, Technik und Medizin. Hannover, 193-267.

Beckmann, K.; Gailing, L.; Hülz, M.; Kemming, H.; Leibenath, M.; Libbe, J.; Stefansky, A. (2013): Räumliche Implikationen der Energiewende. Positionspapier des Raumwissenschaftlichen Netzwerks 11R. Berlin. = Difu-Paper.

BfN - Bundesamt für Naturschutz (Hrsg.) (2010): Bioenergie und Naturschutz. Synergien fördern, Risiken vermeiden. Bonn.

BfN/BBSR - Bundesamt für Naturschutz; Bundesinstitut für Bau-, Stadt- und Raumforschung (Hrsg.) (2011): Kulturlandschaften gestalten! Zum zukünftigen Umgang mit Transformationsprozessen in der Raum- und Landschaftsplanung. Bonn.

BfN/BBSR - Bundesamt für Naturschutz; Bundesinstitut für Bau-, Stadt- und Raumforschung (Hrsg.) (2014a): Den Landschaftswandel gestalten! Potenziale der Landschafts- und Raumplanung zur modellhaften Entwicklung und Gestaltung von Kulturlandschaften vor dem Hintergrund aktueller Transformationsprozesse, Band 1: Bundesweite Übersichten. Bonn.

BfN/BBSR - Bundesamt für Naturschutz; Bundesinstitut für Bau-, Stadt- und Raumforschung (Hrsg.) (2014b): Den Landschaftswandel gestalten! Potentiale der Landschafts- und Raumplanung zu modellhaften Entwicklung und Gestaltung von Kulturlandschaften vor dem Hintergrund aktueller Transformationsprozesse, Band 3: Energiewende als Herausforderung für die Regionen. o. O.

Biesecker, A.; Hofmeister, S. (2006): Die Neuerfindung des Ökonomischen. Ein (re)produktionstheoretischer Beitrag zur Sozialen Ökologie. München.

BMBF - Bundesministerium für Bildung und Forschung (2000): Rahmenkonzept Sozial-Ökologische Forschung. Bonn.

BMBF - Bundesministerium für Bildung und Forschung (2014): BMBF-Fördermaßnahme Umwelt- und gesellschaftsverträgliche Transformation des Energiesystems. http://www.fona.de/ mediathek/pdf/SOEF_Projektblaetter_Energietransformation.pdf (26.02.2015).

BMU - Bundesministerium für Umwelt, Naturschutz und Reaktorsicherheit (2013): Innovation durch Forschung. Jahresbericht 2012 zur Forschungsförderung im Bereich der erneuerbaren Energien. Berlin.

BMVBS - Bundesministerium für Verkehr, Bau und Stadtentwicklung (Hrsg.) (2011): Erneuerbare Energien: Zukunftsaufgabe der Regionalplanung. Bonn.

BMVBS - Bundesministerium für Verkehr, Bau und Stadtentwicklung (Hrsg.) (2013a): Wie kann Regionalplanung zur Anpassung an den Klimawandel beitragen? Bonn. = Forschungen, 157.
BMVBS - Bundesministerium für Verkehr, Bau und Stadtentwicklung (Hrsg.) (2013b): Regionale Energiekonzepte. Vorstellung Modellregionen. Bonn. = MORO-Informationen, 11/1.

BMWi - Bundesministerium für Wirtschaft und Technologie (2011): Forschung für eine umweltschonende, zuverlässige und bezahlbare Energieversorgung. Das 6. Energieforschungsprogramm der Bundesregierung. Berlin.

Brand, K.-W. (2000): Nachhaltigkeitsforschung - Besonderheiten, Probleme und Erfordernisse eines neuen Forschungstypus. In: Brand, K.-W. (Hrsg.): Nachhaltige Entwicklung und Transdisziplinarität. Wissenschaftliche Herausforderung, forschungsmethodische Erfahrungen und forschungspolitische Erfordernisse. Berlin, 9-28.

Brody, A. (2009): Gender and Governance: Overview Report. http:// www.bridge.ids.ac.uk/sites/bridge.ids.ac.uk/files/reports/ Governance_OR_final.pdf. (18.05.2016).

Brownhill, L.; Turner, T. E. (2009): Women and Abuja Declaration for Energy Sovereignity. In: Salleh, Ariel (Hrsg.): Eco-sufficiency \& Global Justice. Women Write Political Ecology. London, 230-250.

Caglar, G.; Castro Varela, M.; Schwenken, H. (Hrsg.) (2012): Geschlecht - Macht - Klima. Feministische Perspektiven auf Klima, gesellschaftliche Naturverhältnisse und Gerechtigkeit, Opladen, Berlin, Toronto.

Clancy, J.; Röhr, U. (2003): Gender and energy: is there a Northern perspective? In: Energy for Sustainable Development, 7, 3, 4449.

DLR - Deutscher Rat für Landespflege (2006): Die Auswirkungen erneuerbarer Energien auf Natur und Landschaft. Meckenheim. = Schriftenreihe des Deutschen Rates für Landespflege, 79.

Eder, K. (1988): Die Vergesellschaftung der Natur: Studien zur sozialen Evolution der praktischen Vernunft. Frankfurt am Main.

Erlinghagen, S. (2014): Demand Response - Ein Modell mit Zukunft für die Schweiz. In: Kawann, C. (Hrsg.): Energie im Wandel. Frauen gestalten die Schweizer Energiewende. Essen, 243-247.

Fainstein, S.; Fainstein, N. (1996): City Planning and Political Values: An Updated View. In: Champbell, S.; Fainstein, S. (Hrsg.): Readings in Planning Theory. Chambridge, Mass, 265-287.

FBW - Forschungsverbund ,Blockierter Wandel?' (Hrsg.) (2007): Blockierter Wandel? Denk- und Handlungsräume für eine nachhaltige Regionalentwicklung. München.

FES - Friedrich-Ebert-Stiftung (Hrsg.) (2008): Klimawandel, Klimaschutz und Gender. Bonn.

Fischer, K. (2011): Genderaspekte der Gebäudekerndämmung aus wiederverwertbaren Rohstoffen. Bremen. = artec-paper, 176.

Fraune, C. (2015): Gender matters: Women, renewable energy, and citizen participation in Germany. In: Energy Research \& Social Science 7, 55-65.

Fürst, D. (2011): Politik und Verwaltung im Wandel. Raumplanung unter veränderten Verhältnissen. In: Akademie für Raumforschung und Landesplanung (ARL) (Hrsg.): Grundrisse der Raumordnung und Raumentwicklung. Hannover, 46-73.

Gailing, L.; Hüesker, F.; Kern, K.; Röhring, A. (2013): Die räumliche Gestaltung der Energiewende zwischen Zentralität und Dezentralität. Explorative Anwendung einer Forschungsheuristik. Erkner. http://www.irs-net.de/download/wp_energiewende_ raum_zentral_dezentral.pdf (10.03.2015). = IRS Working Paper, 51.

Gailing, L.; Leibenath, M. (Hrsg.) (2013): Neue Energielandschaften Neue Perspektiven der Landschaftsforschung. Wiesbaden.

Gailing, L.; Röhring, A. (2015): Was ist dezentral an der Energiewende? Infrastrukturen erneuerbarer Energien als Herausforderungen und Chancen für ländliche Räume. In: Raumforschung und Raumordnung 73, 1, 31-43.

Giris-Musy, G. (2014): Für eine sichere Energieversorgung - Aufgaben und Vorgehen zur wirtschaftlichen Landesversorgung. In: Kawann, C. (Hrsg.): Energie im Wandel. Frauen gestalten die Schweizer Energiewende. Essen, 125-132. 
Hanson, S. (2010): Gender and mobility: new approaches for informing sustainability. In: Gender, Place and Culture 17, 1, 5-23.

Hanusch, M.; Einig, K.; Peters, W.; Schicketanz, S. (2012): Wie kann der Netzausbau raumverträglich(er) gestaltet werden. In: uvp-report 26, 3-4, 148-155.

Hemmati, M.; Röhr, U. (2009): Engendering the climate-change negotiations: experiences, challenges, and steps forward. In: Gender \& Development 17, 1, 19-32.

Hirschl, B.; Aretz, A.; Böther, T. (2011): Ökonomischer Nutzen für EE-Kommunen. In: Ökologisches Wirtschaften 26, 3, 19-21.

Hirschl, B.; Salecki, S.; Böther, T.; Heinbach, K. (2011): Wertschöpfungseffekte durch Erneuerbare Energien in Baden-Württemberg. Berlin.

Hofmeister, S. (1989): Stoff- und Energiebilanzen. Zur Eignung des physischen Bilanz-Prinzips als Konzeption der Umweltplanung. Berlin. = Schriftenreihe des Fachbereichs Landschaftsentwicklung, 58.

Hofmeister, S. (2014): Das Leitbild Nachhaltigkeit - Anforderungen an die Raum- und Umweltplanung. In: Heinrichs, H.; Michelsen, G. (Hrsg.): Nachhaltigkeitswissenschaften. Berlin, Heidelberg, 304-316.

Hofmeister, S.; Katz, C. (2011): Naturverhältnisse. Geschlechterverhältnisse. Nachhaltigkeit. In: Groß, M. (Hrsg.): Handbuch Umweltsoziologie. Wiesbaden, 365-398.

Hofmeister, S.; Katz, C.; Mölders, T. (2013a): Grundlegungen im Themenfeld Geschlechterverhältnisse und Nachhaltigkeit. In: Hofmeister, S.; Katz, C.; Mölders, T. (Hrsg.): Geschlechterverhältnisse und Nachhaltigkeit. Die Kategorie Geschlecht in den Nachhaltigkeitswissenschaften. Opladen, Berlin, Toronto, 33-76.

Hofmeister, S.; Katz, C.; Mölders, T. (2013b): Fazit. Die Kategorie Geschlecht: Neue Perspektiven für die Nachhaltigkeitswissenschaften. In: Hofmeister, S.; Katz, C.; Mölders, T. (Hrsg.): Geschlechterverhältnisse und Nachhaltigkeit. Die Kategorie Geschlecht in den Nachhaltigkeitswissenschaften. Opladen, Berlin, Toronto, 339-351.

Hofmeister, S.; Mölders, T. (2006): Geschlecht als Basiskategorie der Nachhaltigkeitsforschung. In: Schäfer, M.; Schultz, I.; Wendorf, G. (Hrsg.): Gender-Perspektiven in der Sozial-ökologischen Forschung. Herausforderungen und Erfahrungen aus inter- und transdisziplinären Projekten. München, 17-37.

Hofmeister, S.; Scurrell, B. (2006): Annäherungen an ein sozial-ökologisches Raumkonzept. In: GAIA 15, 4, 275-284.

Hofmeister, S.; Scurrell, B. (2016): Die „Energielandschaft“ als StadtLandschaft. Die Transformationsgeschichte einer Region in sozial-ökologischer Perspektive. In: Hofmeister, S.; Kühne, O. (Hrsg.): StadtLandschaften. Die neue Hybridität von Stadt und Land. Wiesbaden. (i. D.)

Holland-Cunz, B. (2014): Die Natur der Neuzeit. Eine feministische Einführung. Opladen, Berlin, Toronto.

Hudson, C. (2012): Governing the city - Gender and urban governance. In: Genderarchland (Hrsg.): Theorizing and Practising Gender Sensitive Planning in European Discourse. Documentation. Scientific conference with integrated meeting of the European network „Gender, Diversity and Urban Sustainability (GDUS)“, 25.-28. April 2012. Hannover, 57-58.

Hübner, G.; Müller, M.; Röhr, U. (o. J.): Erneuerbare Energien und Ökostrom - zielgruppenspezifische Kommunikationsstrategien. Abschlussbericht zum BMU-Verbundprojekt (FKZ 0325107/8), http://www.genanet.de/fileadmin/user_upload/dokumente/GenderKlima-Energie/Oekostrom_kurzfassung.pdf (21.01.16).

IRS - Leibniz Institut für Regionalentwicklung und Strukturplanung (Hrsg.) (o. J.): Forschungsprogramm 2015-2018. http://www.irsnet.de/download/forschung/IRSFP1518.pdf (27.02.15).

Kanning, H. (2005): Brücken zwischen Ökologie und Ökonomie Umweltplanerisches und ökonomisches Wissen für ein nachhaltiges regionales Wirtschaften. München.

Kanning, H. (2013): Kommentar: Genderspezifische Ansätze zur Energiewende. In: Hofmeister, S.; Katz, C.; Mölders, T. (Hrsg.):
Geschlechterverhältnisse und Nachhaltigkeit. Die Kategorie Geschlecht in den Nachhaltigkeitswissenschaften. Berlin, Toronto, 262-266.

Kanning, H.; Buhr, N.; Steinkraus, K. (2009): Erneuerbare Energien - Räumliche Dimensionen, neue Akteurslandschaften und planerische (Mit)Gestaltungspotenziale am Beispiel des Biogaspfades. In: Raumforschung und Raumordnung 67, 2, 142-156.

Katz, C.; Mölders, T. (2013): Schutz, Nutzung und nachhaltige Gestaltung - Geschlechteraspekte im Umgang mit Natur. In: Hofmeister, S.; Katz, C.; Mölders, T. (Hrsg.): Geschlechterverhältnisse und Nachhaltigkeit. Die Kategorie Geschlecht in den Nachhaltigkeitswissenschaften. Berlin, Toronto, 269-277.

Klagge, B. (2013): Governance-Prozesse für erneuerbare Energien Akteure, Koordinations- und Steuerungsstrukturen. In: Akademie für Raumforschung und Landesplanung (ARL) (Hrsg.): Governance-Prozesse für erneuerbare Energien. Hannover, 7-16. = Arbeitsberichte der ARL, 5 .

Klagge, B.; Arbach, C.; Franck, E.; Heidrich, B.; Hirschfeld, M.; Liebrenz, F.; Pohl, M.; Sünnemann, A.; Thom, S.; Wotha, B. (2013): Empfehlungen für die Weiterentwicklung der Koordinations- und Steuerungsstrukturen zum Ausbau der erneuerbaren Energien in Deutschland. In: Akademie für Raumforschung und Landesplanung (ARL) (Hrsg.): Governance-Prozesse für erneuerbare Energien. Hannover, 1-6. = Arbeitsberichte der ARL, 5.

Klinger, C.; Knapp, G.-A.; Sauer, B. (Hrsg.) (2007): Achsen der Ungleichheit. Zum Verhältnis von Klasse, Geschlecht und Ethnizität. Frankfurt am Main, New York.

Knothe, B. (2013): Zwischen Identität und Dekonstruktion - Wasserwirtschaftliche Infrastruktur aus der Perspektive von Gender und Intersektionalität. In: Hofmeister, S.; Katz, C.; Mölders, T. (Hrsg.): Geschlechterverhältnisse und Nachhaltigkeit. Die Kategorie Geschlecht in den Nachhaltigkeitswissenschaften. Opladen, Berlin, Toronto, 253-262.

Kropp, C. (2002): „Natur“. Soziologische Konzepte. Politische Konsequenzen. Opladen.

Kruse, S. (2010): Vorsorgendes Hochwassermanagement im Wandel. Ein sozial-ökologisches Raumkonzept für den Umgang mit Hochwasser. Wiesbaden.

Lambrou, Y.; Piana, G. (2006): Energy and Gender Issues in rural sustainable development. Rome. http://www.fao.org/docrep/010/ ai021e/ai021e00.htm. (27.02.2015).

Massey, D. (1994): Space, place and gender. Minneapolis.

Moser, P. (2011): Wegweiser für eine dezentrale Energieversorgung. In: Ökologisches Wirtschaften 26, 3, 16-8.

Moss, T.; Gailing, L.; Kern, K.; Naumann, M.; Röhring, A. (2013): Energie als Gemeinschaftsgut? Anregungen für die raumwissenschaftliche Energieforschung. Erkner. http://www.irs-net.de/ download/wp_gemeinschaftsgut_energie.pdf (23.01.2016). = IRS Working Paper, 50.

Partzsch, D. (1970): Daseinsgrundfunktionen, I. Die Raumansprüche der Funktionsgesellschaft. In: Akademie für Raumforschung und Landesplanung (Hrsg.): Handwörterbuch der Raumforschung und Raumordnung, I. Hannover, 424-430.

Poferl, A. (2001): Doing Gender, Doing Nature? Einführende Bemerkungen zur Intention des Bandes. In: Nebelung, A.; Poferl, A.; Schultz, I. (Hrsg.): Geschlechterverhältnisse - Naturverhältnisse. Feministische Auseinandersetzungen und Perspektiven der Umweltsoziologie. Opladen, 9-17.

Radtke, J. (2014): Die Energiewende in Deutschland und die Partizipation der Bürger. In: BBE-Newsletter 2/2014. http://www.bb-e.de/fileadmin/inhalte/aktuelles/2014/02/NL02_Gastbeitrag _ Radtke.pdf. (02.01.2016).

Radtke, J. (2015): Das Zusammenspiel von Raum und Technik bei der Etablierung Erneuerbarer Energien. Transformationen in der Energiewende. In: Raumforschung und Raumordnung 73, 6, 389-405. 
Röhr, U. (2001): Gender \& Energie. Aus der Sicht des Nordens. Background Paper für den Workshop „Gender Perspectives for Earth Summit 2002: Energy, Transport, Information for Decision-Making“, 10.-12. Januar 2001, Berlin. http://www.genanet. de/fileadmin/user_upload/dokumente/Themen/Energie/gender_ energy_de.PDF (13.01.2016).

Röhr, U.; Alber, G.; Kösters, J. (2012): Erneuerbare Energien und Ökostrom - zielgruppenspezifische Kommunikationsstrategien. Modul II Determinanten der Wechselbereitschaft von Frauen zu Ökostrom. Abschlussbericht zum BMU-Verbundprojekt (FKZ: 0325108). Berlin. http://www.genanet.de/fileadmin/user_upload/ dokumente/Gender-Klima-Energie/AbschlussberichtFKZ_0325108nbf.pdf (23.01.2016).

Ryan, S. E. (2014): Rethinking gender and identity in energy studies. In: Energy Research \& Social Science, 1, 96-105.

Schmitt, M. (2005): Rurale Frauen- und Geschlechterforschung. In: Beetz, St.; Brauer, K.; Neu, C. (Hrsg.): Handwörterbuch zur ländlichen Gesellschaft in Deutschland. Wiesbaden, 210-217.

Schot, J.; Geels, F. W. (2007): Niches in evolutionary theories of technical change. In: Journal of Evolutionary Economics 17, 5, 605-622.

Schultz, I.; Hummel, D.; Hayn, D. (2006): Geschlechterverhältnisse. In: Becker, E.; Jahn, T. (Hrsg.): Soziale Ökologie. Grundzüge einer Wissenschaft von den gesellschaftlichen Naturverhältnissen. Frankfurt am Main, New York, 224-235.

Schultz, I.; Schramm, E.; Hummel, D. (2015): Gender als Integrationsdimension in der transdisziplinären SÖF (sozial-ökologischen Forschung). In: Katz, C.; Heilmann, S.; Thiem, A.; Koch, L. M.; Moths, K.; Hofmeister, S. (Hrsg.): Nachhaltigkeit anders denken. Veränderungspotenziale durch Geschlechterperspektiven. Wiesbaden, 217-230.

Schultz, I.; Stieß, I. (2009): Gender aspects of sustainable consumption strategies and instruments. Eupopp (Policies to Promote Sustainable Consumption Patterns) Working Paper 1. Frankfurt am Main. http://www.eupopp.net/docs/isoe-gender_wp1_20090426-endlv. pdf (13.01.2016).

Schweizer-Ries, P.; Rau, I.; Hildebrand, J. (2011): Akzeptanz- und Partizipationsforschung zur Energienachhaltigkeit. In: Transformationsforschung für ein nachhaltiges Energiesystem. Beiträge zur FVEE-Jahrestagung 2011. Berlin, 138-144. http://www.fvee. de/fileadmin/publikationen/Themenhefte/th2011-2/th2011_07_ 01.pdf (13.05.2016)

Smith, A.; Stirling, A.; Berkhout, F. (2005): The governance of sustainable socio-technical transition. In: Research Policy 34, 10, 1491-1510.

SRU - Sachverständigenrat für Umweltfragen (2008): Umweltschutz im Zeichen des Klimawandels. Umweltgutachten. Berlin.

SRU - Sachverständigenrat für Umweltfragen (2011): Wege zur 100\% erneuerbaren Stromversorgung. Sondergutachten. Berlin.

Voß, J.-P.; Bornemann, B. (2011): The Politics of reflexive governance. Challenges for designing Adaptive Management and Transition Management. In: Ecology \& Society 16, 2, 9.
Voß, J.-P.; Smith, A.; Grin, J. (2009): Designing long-term policy: rethinking transition management. In: Policy Sciences 42, 4, 275-302.

Wastl-Walter, D. (2010): Gender Geographien. Geschlecht und Raum als soziale Konstruktionen. Stuttgart.

WBGU - Wissenschaftlicher Beirat der Bundesregierung globale Umweltveränderungen (2011): Hauptgutachten. Welt im Wandel. Gesellschaftsvertrag für eine Große Transformation. Berlin.

Weller, I. (2004): Nachhaltigkeit und Gender. Neue Perspektiven für die Gestaltung und Nutzung von Produkten. München.

Weller, I. (2012): Klimawandel, Konsum und Gender. In: Caglar, G.; Castro Varela, M.; Schwenken, H. (Hrsg.): Geschlecht - Macht Klima. Feministische Perspektiven auf Klima, gesellschaftliche Naturverhältnisse und Gerechtigkeit. Opladen, Berlin, Toronto, 177-190.

Weller, I. (2013): Nachhaltiger Konsum, Lebensstile und Geschlechterverhältnisse. In: Hofmeister, S.; Katz, C.; Mölders, T. (Hrsg.): Geschlechterverhältnisse und Nachhaltigkeit. Die Kategorie Geschlecht in den Nachhaltigkeitswissenschaften. Berlin, Toronto, 286-296.

West, C.; Zimmerman, D. H. (1987): Doing Gender. In: Gender \& Society $1,2,125-151$.

Wichterich, C. (2002): Sichere Lebensgrundlagen statt effizienterer Naturbeherrschung - Das Konzept nachhaltige Entwicklung aus feministischer Sicht. In: Görg, C.; Brand, U. (Hrsg.): Mythen globalen Umweltmanagements. Rio +10 und die Sackgassen „,nachhaltiger Entwicklung“. Münster, 72-91.

Wichterich, C. (2015): Livelihood, Ökonomisierung des Lebendigen und gutes Leben - Feministische Perspektiven auf die verschlungenen Wege von Rio 1992 nach Rio 2012. In: Katz, C.; Heilmann, S.; Thiem, A.; Moths, K.; Koch, L. M.; Hofmeister, S. (Hrsg.): Nachhaltigkeit anders denken. Veränderungspotenziale durch $\mathrm{Ge}$ schlechterperspektiven. Wiesbaden, 31-42.

Wiehe, J.; Buhr, N.; Wolf, U.; Kanning, H.; Rode, M. (2010): Planerische Koordinierung für einen natur- und raumverträglichen Ausbau energetischer Biomassepfade. In: Rode, M.; Kanning, H. (Hrsg.): Natur- und raumverträglicher Ausbau energetischer Biomassepfade. Stuttgart, 241-251.

Wiliarty, S. E. (2011): Gender and energy policy making under the first Merkel government. In: German Politics 20, 3, 449-463.

Winterfeld, U. von (2012): Partizipieren reicht nicht. Klimawandel und demokratische Kultur. http://edoc.sub.uni-hamburg.de/ klimawandel/files/779/dynaklim_Pub32_032.pdf (18.05.2016). = dynaklim-Publikation, 32.

Winterfeld, U. von (2013): Bedeutet „Governance“ Partizipation - und Partizipation „Empowerment“"? In: Hofmeister, S.; Katz, C.; Mölders, T. (Hrsg.): Geschlechterverhältnisse und Nachhaltigkeit. Die Kategorie Geschlecht in den Nachhaltigkeitswissenschaften. Berlin, Toronto, 320-329.

Zoellner, J.; Rau, I.; Schweizer-Ries, P. (2011): Beteiligungsprozesse und Entwicklungschancen für Kommunen und Regionen. In: Ökologisches Wirtschaften 26, 3, 25-27. 